\title{
ON THE GENERALIZED INPUT ESTIMATION
}

\author{
Vesselin JILKOV and Xiao RONG LI
}

\section{Introduction}

Consider the problem of maneuvering target tracking within the framework of the familiar time invariant linear dynamic system ${ }^{1}$

$$
\begin{aligned}
x_{k+1} & =F x_{k}+G u_{k}+w_{k} \\
z_{k+1} & =H x_{k+1}+v_{k+1},
\end{aligned} \quad k=0,1,2, \ldots
$$

where $x_{k} \in \mathbf{R}^{n_{x}}$ denotes the target state with transition matrix $F, u_{k} \in \mathbf{R}^{n_{u}}$ is the control input with transition matrix $G, z_{k} \in \mathbf{R}^{n_{z}}$ is the measurement with measurement matrix $H$, and $w_{k} \in \mathbf{R}^{n_{x}}, v_{k} \in \mathbf{R}^{n_{z}}$ denote respectively the process noise and measurement errors which are assumed independent Gaussian white noises with zero means and covariances $Q_{k}$ and $R_{k}$.

The classical input estimation (IE) ${ }^{2}$ assumes that the unknown control input is constant, i.e. if the maneuver has started at time $k$, then

$$
u_{j}= \begin{cases}0 & \text { for } j=0,1, \ldots, k-1 \\ u & \text { for } j=k, \ldots, k+N-1,\end{cases}
$$

where $N$ denotes the detection window length. This assumption allows to use the least squares (LS) method for parameter estimation to obtain an estimate of the input $u$ over the interval $[k, k+N)$, based on the information contained in the innovations of the Kalman filter assuming zero-input in the interval $[0, k+N)$.

In order to relax this restrictive and unrealistic assumption it was suggested ${ }^{3}$ to represent the unknown input $u_{k}$ as a linear combination of time functions, viz.

$$
u_{k}=\sum_{l=1}^{p} a_{l} b_{l}\left(t_{k}\right),
$$

where $b_{l}\left(t_{k}\right)$ are known scalar functions of time and $a_{l}$ are unknown constant vector coefficients. For the so defined "non-constant" input the LS estimation technique has been applied and a thorough algorithm derivation has been performed. ${ }^{3}$

INFORMATION \& SECURITY. An International Journal, Vol. 9, 2002, 107-113 
Apparently, if we consider the input transition matrix $G$ in (1) as time invariant then the presentation of the input (4) is more general than that of the constant input $u_{k}=$ $u$ in (3). On the other hand, however, if we consider the overall unknown input $a \triangleq\left(a_{1}, a_{2}, \ldots, a_{p}\right)$, it is in fact constant and the known time functions $b_{l}\left(t_{k}\right), l=$ $1, \ldots, p$ influence this input as transition coefficients (in the same manner as the input transition matrix $G$ does). That is why it is more natural that these coefficients be attributed to the input transition (coefficient) matrix rather than to the input itself. This underlying reason has led us to the following two observations.

- The generalized IE model (1) with (4) is a particular case of the constant input model

$$
x_{k+1}=F x_{k}+G_{k} u+w_{k}
$$

with time-varying input transition matrix $G_{k}$. Indeed, if we set

$$
a \triangleq\left[a_{1}^{\prime} \ldots a_{p}^{\prime}\right]^{\prime} \text { and } G_{k}^{a} \triangleq\left[b_{1}\left(t_{k}\right) G\left|b_{2}\left(t_{k}\right) G\right| \ldots \mid b_{p}\left(t_{k}\right) G\right]
$$

then (1) with (4) can be recast as

$$
x_{k+1}=F_{k} x_{k}+G_{k}^{a} a+w_{k} .
$$

That is, $a$ stands for the unknown constant input and $G_{k}^{a}$ for the known (timevarying) input transition matrix. Of course (5) comprises (7) and is not restricted to the particular choice of $G_{k}$ as $G_{k}^{a}$.

- The classical IE for constant input is valid for time-varying systems, and in particular for (5) (respectively (7)).

These observations imply that the GIE of ${ }^{3}$ is a particular case of the classical IE with time-varying input transition matrix $G_{k}$ (if we set $G_{k}=G_{k}^{a}$ ). Next, we describe in more details the IE for time-varying systems and show how the GIE can be obtained from it.

\section{IE for Time-Varying Systems}

Although the IE method of Chan, Hu and Plant, ${ }^{2}$ has been traditionally treated in time invariant system setting, it is valid for time-varying systems as well and no additional difficulties appear in this consideration. We summarize the basic IE method with reference to the target model (5).

The optimal Kalman filter (KF) for the system (5), (2), where $F_{k}$ may be also time- 
varying, is ${ }^{4}$

$$
\begin{aligned}
\hat{x}_{k+1} & =\left(I-K_{k+1} H\right) F_{k} \hat{x}_{k}+\left(I-K_{k+1} H\right) G_{k} u_{k}+K_{k+1} z_{k+1} \\
P_{k+1} & =\left(I-K_{k+1} H\right)\left(F_{k} P_{k} F_{k}^{\prime}+Q_{k}\right) \\
K_{k+1} & =\left(F_{k} P_{k} F_{k}^{\prime}+Q_{k}\right) H^{\prime}\left[H\left(F_{k} P_{k} F_{k}^{\prime}+Q_{k}\right) H^{\prime}+R_{k+1}\right]^{-1} .
\end{aligned}
$$

Let the assumption (3) holds and denote by $\hat{x}_{j}^{*}$ and $\hat{x}_{j}$ the estimates of the hypothetical Kalman filter with the correct input $u_{j}$ of (3), and the real KF, running with the zeroinput model $u_{j}=0, j=k, \ldots, k+N-1$, respectively. Their residuals, defined respectively as

$$
\tilde{z}_{j}^{*} \triangleq z_{j}-H \hat{x}_{j}^{*}, \quad \tilde{z}_{j} \triangleq z_{j}-H \hat{x}_{j}, \quad j=1,2, \ldots
$$

satisfy

$$
\tilde{z}_{k+n}=H D_{k+n} u+\tilde{z}_{k+n}^{*}, \quad n=1,2, \ldots, N
$$

where

$$
D_{k+n} \triangleq \sum_{i=1}^{n} \prod_{j=n}^{i+1}\left(I-K_{k+j} H\right) F_{k+j}\left(I-K_{k+i} H\right) G_{k+i-1} .
$$

It is known that $\left\{\tilde{z}_{k+n}^{*}\right\}_{n=1,2, \ldots, N}$ is a white noise sequence with $\tilde{z}_{k+n}^{*} \sim \mathcal{N}\left(0, S_{k+n}\right)$, where the covariance $S_{k+n}=H P_{k+n} H^{\prime}+R_{k+n} .{ }^{4}$ Thus according to (12) the residuals of the real (zero-input) KF provide noisy measurements of the unknown input, and the best linear unbiased estimate (BLUE) of $u$ can be straightforwardly obtained by means of the LS method for this system. ${ }^{4}$

Specifically, the system (12) can be recast in the "batch form"

$$
\tilde{\mathcal{Z}}=\mathcal{H} u+\tilde{\mathcal{Z}}^{*},
$$

where stacked vectors and matrices are denoted as follows

$$
\begin{gathered}
\tilde{\mathcal{Z}}=\left[\begin{array}{lll}
\tilde{z}_{k+1}^{\prime} & \ldots & \tilde{z}_{k+N}^{\prime}
\end{array}\right]^{\prime}, \\
\mathcal{H}=\left[\begin{array}{ccc}
\left(H D_{k+1}\right)^{\prime} & \ldots & \left(H D_{k+N}\right)^{\prime}
\end{array}\right]^{\prime}, \\
\tilde{\mathcal{Z}}^{*}=\left[\begin{array}{lll}
\tilde{z}_{k+1}^{* \prime} & \ldots & \tilde{z}_{k+N}^{* \prime}
\end{array}\right]^{\prime}
\end{gathered}
$$

and $\tilde{\mathcal{Z}}^{*} \sim \mathcal{N}(0, \mathcal{S})$ for $\mathcal{S}=$ block-diag $\left\{S_{k+1}, \quad \ldots, \quad S_{k+N}\right\}$. Then the BLUE of $u$ which minimizes the normalized error

$$
\mathcal{L}_{\mathrm{LS}}(u) \triangleq \tilde{\mathcal{Z}}^{* \prime} \mathcal{S}^{-1} \tilde{\mathcal{Z}}^{*}=(\tilde{\mathcal{Z}}-\mathcal{H} u)^{\prime} \mathcal{S}^{-1}(\tilde{\mathcal{Z}}-\mathcal{H} u)
$$


is

$$
\hat{u}=\mathcal{P} \mathcal{H}^{\prime} \mathcal{S}^{-1} \tilde{\mathcal{Z}} \text { with covariance } \mathcal{P}=\left(\mathcal{H}^{\prime} \mathcal{S}^{-1} \mathcal{H}\right)^{-1}
$$

The minimal normalized error is given by

$$
\hat{\mathcal{L}}_{\mathrm{LS}} \triangleq \mathcal{L}_{\mathrm{LS}}(\hat{u})=(\tilde{\mathcal{Z}}-\mathcal{H} \hat{u})^{\prime} \mathcal{S}^{-1}(\tilde{\mathcal{Z}}-\mathcal{H} \hat{u})=\tilde{\mathcal{Z}}^{\prime} \mathcal{S}^{-1} \tilde{\mathcal{Z}}-\Delta \mathcal{L}_{\mathrm{LS}}(\hat{u})
$$

with

$$
\Delta \mathcal{L}_{\mathrm{LS}}(\hat{u})=\hat{u}^{\prime} \mathcal{P}^{-1} \hat{u}=\left(\mathcal{H}^{\prime} \mathcal{S}^{-1} \tilde{\mathcal{Z}}\right)^{\prime} \mathcal{P}\left(\mathcal{H}^{\prime} \mathcal{S}^{-1} \tilde{\mathcal{Z}}\right)
$$

and $\Delta \mathcal{L}_{\mathrm{LS}}(\hat{u})$ is $\chi_{n_{u}}^{2}$ distributed, provided the true input $u$ is zero. ${ }^{1}$

Thus, the first stage of the common IE method - estimation of the input is performed via (17). The second stage - maneuver detection - realizes the significance test ${ }^{4}$

$$
\Delta \mathcal{L}_{\mathrm{LS}}>\lambda
$$

through (19), where $\lambda$ is chosen for a given $P_{\text {FA }}$. The third stage of the IE algorithm estimate correction - is performed in case of detecting a maneuver according to

$$
\begin{aligned}
\hat{x}_{k+N}^{u} & =\hat{x}_{k+N}+\underbrace{D_{k+N} \hat{u}}_{\text {correction term }} \\
P_{k+N}^{u} & =P_{k+N}+\underbrace{D_{k+N} \mathcal{P} D_{k+N}^{\prime}}_{\text {uncertainty increase }}
\end{aligned}
$$

In the above, we very briefly recalled the known IE method with the sole difference of considering the generic time-varying target model.

\section{GIE as a Corollary of IE}

Now that the IE is available one can obtain the GIE algorithm of ${ }^{3}$ (specifically, the results presented in sections III and IV therein) as a corollary of the above given common IE. Although it should be apparent from the two remarks made in the Introduction we illustrate some details.

Consider the problem as formulated by Lee and Tahk.${ }^{3}$ Let us set for this problem $a$ and $G_{k}^{a}$ as in (6) and substitute $G_{k}$ with $G_{k}^{a}$ and $u$ with $a$ throughout in the Eqns (13) (22) of the IE.

After some routine formulae manipulations the following key intermediate relations can be subsequently obtained 


$$
\begin{aligned}
& D_{k+n}= \\
& \sum_{i=1}^{n} M_{k+n}^{k+i}\left(I-K_{k+i} H\right) G_{k+i-1}= \\
& \sum_{i=1}^{n} M_{k+n}^{k+i}\left(I-K_{k+i} H\right)\left[b_{1}\left(t_{k+i-1}\right) G|\ldots| b_{p}\left(t_{k+i-1}\right) G\right]= \\
& {\left[\sum_{i=1}^{n} M_{k+n}^{k+i}\left(I-K_{k+i} H\right) N_{k+i} b_{1}\left(t_{k+i-1}\right)|\ldots| \sum_{i=1}^{n} M_{k+n}^{k+i}\left(I-K_{k+i} H\right) N_{k+i} b_{p}\left(t_{k+i-1}\right)\right]}
\end{aligned}
$$

where all quantities $M_{k+n}^{k+i}, N_{k+i}, D_{k+n}^{l}, C_{k+n}^{l}, A_{k+n}^{k+i}, \Omega, \Gamma$ are the same as defined by Lee and Tahk. ${ }^{3}$

Consider now the IE algorithm. Firstly, the error (16) is

$$
\mathcal{L}_{\mathrm{LS}}(u)=\sum_{n=1}^{N}\left(\tilde{z}_{k+n}-H D_{k+n} u\right)^{\prime} S_{k+n}^{-1}\left(\tilde{z}_{k+n}-H D_{k+n} u\right)
$$

and after the substitution of $G_{k} u$ with $G_{k}^{a} a$, in view of (28), it transforms to the performance index $L(k, N)$ defined in ${ }^{3}$ through the identity $-\frac{1}{2} \mathcal{L}_{\mathrm{LS}}(u)=L(k, N)$ (as in Eqn. (12) of ${ }^{3}$ ). Secondly, the IE equation (17) after the substitutions (29), (30) leads to the basic GIE equation (20) of ${ }^{3}$, since

$$
\mathcal{H}^{\prime} \mathcal{S}^{-1} \tilde{\mathcal{Z}}=\Omega \text { and } \mathcal{P}^{-1} \triangleq \mathcal{H}^{\prime} \mathcal{S}^{-1} \mathcal{H}=\Gamma .
$$


Further, in view of (32), Lema 1, Lema 2, and the maneuver detector ((28) of $\left.{ }^{3}\right)$ immediately follow from (18), (19), and (20) respectively. Finally, (21) and (22) yield the correction equations (30) and (31) of ${ }^{3}$, respectively, that can be seen by accounting for (24).

Thus we proved that the GIE algorithm of ${ }^{3}$ can be obtained from the common IE algorithm applied to the particular choice of $G_{k}$ as $G_{k}^{a}$, and $u$ as $a$.

\section{Conclusion}

More insight has been given to the problem of input estimation. It has been shown that the so called generalized input estimation can be interpreted as a particular case of the conventional input estimation with constant input and time-varying transition matrix of the input. The latter setting, however, is more general than that of the generalized input estimation. In practice, it enables designing models with various "non-constant" inputs to be done through the design of the input transition matrix.

\section{Acknowledgement}

Research supported by ONR grant N00014-00-1-0677, NSF grant ECS-9734285, and NASA/LEQSF grant (2001-4)-01.

\section{Notes:}

1 Xiao Rong Li and Vesselin Jilkov, "A Survey of Maneuvering Target Tracking-Part IV: Decision-Based Methods," in Proc. SPIE Conf. Signal and Data Processing of Small Targets, vol. 4728 (Orlando, Florida: April 2002).

2 Y. T. Chan, A. G. C. Hu, and J. B. Plant, "A Kalman Filter Based Tracking Scheme with Input Estimation," IEEE Trans. AES, AES-15, 2, (Mar. 1979): 237-244.

${ }^{3}$ H. Lee and M.-J. Tahk, "Generalized input-estimation technique for tracking maneuvering targets," IEEE Transactions on Aerospace and Electronic Systems 35, 4 (1999): $1388-1402$.

4 Yaakov Bar-Shalom, Xiao Rong Li, and T. Kirubarajan, Estimation with Applications to Tracking and Navigation: Theory, Algorithms, and Software (New York: Wiley, 2001). 
VESSELIN PETROV JILKOV received an M.S. degree in Mathematics from the University of Sofia, Bulgaria, in 1982, a Ph.D. degree in Electrical Engineering in 1988, and the academic rank of Associate Research Professor at the Bulgarian Academy of Sciences (BAS) in 1997. He was a research scientist with the RD Institute of Special Electronics (1982-1988), and with the Central Laboratory for Parallel Processing - BAS, Sofia (1989-1999), respectively. There, he worked as a key researcher in numerous academic and industry projects (Bulgarian and international) in the areas of Kalman filtering, multi-target tracking, multi-sensor data fusion, and parallel processing. Since 1999 Dr. Jilkov has been with the Department of Electrical Engineering, University of New Orleans, New Orleans, USA, teaching and conducting research in the areas of hybrid systems estimation and target tracking. Dr. Jilkov is author and coauthor of over 10 journal and 25 conference papers. He is a member of IEEE and ISIF. Dr. Jilkovs main research interests include stochastic systems, applied estimation and filtering, target tracking, multi-sensor data fusion, and parallel processing.

XIAO RONG LI received a B.S. degree and an M.S. degree from Zhejiang University, Hangzhou, Zhejiang, PRC, in 1982 and 1984, respectively, and an M.S. degree and a Ph.D. degree from University of Connecticut in 1990 and 1992, respectively. He joined Department of Electrical Engineering, University of New Orleans in 1994, where he is currently Professor and Director of Information and Systems Laboratory. During 1986-1987 he did research on electric power at University of Calgary, Canada. He was an Assistant Professor at University of Hartford from 1992 to 1994. Professor Li has authored or coauthored four books: Estimation and Tracking, Multitarget-Multisensor Tracking, Probability, Random Signals, and Statistics, and Estimation with Applications to Tracking and Navigation, four book chapters, more than thirty journal articles, more than 110 conference papers. Dr. Li has received a CAREER award and an RIA award from the National Science Foundation; served as an Editor for IEEE Transactions on Aerospace and Electronic Systems since 1996, an Associate Editor, 1995-1996, an Editor for Communications in Information and Systems since 2000, Vice President for Conferences and Technical Activities and member of Board of Directors, International Society of Information Fusion since 1998; served as General Chair for 2002 International Conference on Information Fusion; and won several outstanding paper awards. Dr. Li's current research interests include signal and data processing, information fusion, target tracking, stochastic systems, statistical inference, and electric power. 